\title{
Adaptive Equalizer Using Selective Partial Update Algorithm and Selective Regressor Affine Projection Algorithm over Shallow Water Acoustic Channels
}

\author{
Masoumeh Soflaei and Paeiz Azmi \\ Faculty of Electrical and Computer Engineering, Tarbiat Modares University, P.O. Box 14115-194, Tehran, Iran \\ Correspondence should be addressed to Paeiz Azmi; pazmi@modares.ac.ir
}

Received 4 October 2012; Accepted 19 November 2012; Published 25 May 2014

Academic Editor: Hamid Ahmadian

Copyright (C) 2014 M. Soflaei and P. Azmi. This is an open access article distributed under the Creative Commons Attribution License, which permits unrestricted use, distribution, and reproduction in any medium, provided the original work is properly cited.

\begin{abstract}
One of the most important problems of reliable communications in shallow water channels is intersymbol interference (ISI) which is due to scattering from surface and reflecting from bottom. Using adaptive equalizers in receiver is one of the best suggested ways for overcoming this problem. In this paper, we apply the family of selective regressor affine projection algorithms (SR-APA) and the family of selective partial update APA (SPU-APA) which have low computational complexity that is one of the important factors that influences adaptive equalizer performance. We apply experimental data from Strait of Hormuz for examining the efficiency of the proposed methods over shallow water channel. We observe that the values of the steady-state mean square error (MSE) of SR-APA and SPU-APA decrease by $5.8(\mathrm{~dB})$ and $5.5(\mathrm{~dB})$, respectively, in comparison with least mean square (LMS) algorithm. Also the families of SPU-APA and SR-APA have better convergence speed than LMS type algorithm.
\end{abstract}

\section{Introduction}

Underwater acoustic communications suffer from doppler shifts, noise, scattering from surface, and reflecting from bottom that cause multipath spread especially in shallow water channels. Multipath spread causes intersymbol interference (ISI), which influences reliable transmission in underwater acoustic communications. One of the best ways suggested for overcoming this effect is using adaptive equalization methods in receivers. Some factors such as adaptive filter and adaptive algorithms influence this method, so it is important to choose adaptive algorithms with low computational complexity and more ability for tracking changes in channel.

Least mean square (LMS) algorithm is the most popular because of its simplicity in computations and implementation that is used by Stojanovic et al. $[1,2]$ in underwater acoustic communication. Recursive least square (RLS) due to the fastest convergence speed is the best adaptive filter algorithm used by Zheng et al. [3] and Freitag et al. [4] in this channel in spite of its computational complexity. Computational complexity in some algorithms such as RLS is one of the main problems, so some other algorithms such as selective regressor affine projection algorithm (SR-APA) and selective partial update (SPU) that have good tradeoff between computational complexity and convergence speed are suggested. In SPU algorithm, the blocks of filter coefficients have been updated in every iteration that is selected by special criteria. Important examples of this algorithm are different types of selective partial update normalized least mean square (SPUNLMS) [5-7] such as MAX-NLMS [8], N-MAX NLMS [9] (the number of filter coefficients to be updated is N), and the family of SPU affine projection algorithms (SPU-APA) [10]. In selective regressor algorithm approach, a subset of input regressors should be selected by selection criteria in every iteration. In comparison with LMS, the value of the steadystate mean square error (MSE) of SR-APA by selecting 3 of 4 input regressors decreases by $5.8(\mathrm{~dB})$ and the value of the steady-state MSE of SPU-APA by selecting 3 of 4 blocks of filter coefficients decreases by $5.5(\mathrm{~dB})$.

In this paper, our objective is to apply SPU-APA and SRAPA algorithms that have a good tradeoff between convergence speed and computational complexity for underwater 
acoustic communications. Also, we show the result of using the performance of these algorithms in comparison with RLS and NLMS.

This paper is organized as follows: in the next section, we present the basis of APA, SR-APA, and SPU-APA. In Section 3, we introduce a model for shallow water channel. Section 4 presents the computational complexity of different algorithms that are introduced. We show the simulation results in Section 5, and we conclude the paper in Section 6.

\section{Basis of Affine Projection Algorithm, Selective Regressor Affine Projection Algorithm (SR-APA), and Selective Partial Update Affine Projection Algorithm (SPU-APA)}

2.1. APA. In this paper, $x, d$, and $e$ denote input, desired, and output error signals, respectively. $\mathbf{h}$ is the filter coefficients vector with order $M \times 1$ and $\mu$ is the step size. The family of affine projection algorithms (APA) is derived by solving

$$
\operatorname{Min}\|\mathbf{h}(n+1)-\mathbf{h}(n)\|^{2}
$$

subject to $\mathbf{d}(n)=\mathbf{X}^{T}(n) \mathbf{h}(n+1)$. Lagrange multipliers method leads to the following recursion:

$$
\mathbf{h}(n+1)=\mathbf{h}(n)+\mu \mathbf{C}(n) \mathbf{X}(n) \mathbf{W}(n) \mathbf{e}(n),
$$

where $\mathbf{e}(n)=\mathbf{d}(n)-\mathbf{X}^{T}(n) \mathbf{h}(n)$.

$\mathbf{h}$ is the $M \times 1$ column vector of filter coefficients, $\mathbf{X}$ is the $M \times P$ matrix of the input signal $\mathbf{X}(n)=[\mathbf{x}(n), \mathbf{x}(n-$ $D), \ldots, \mathbf{x}(n-(P-1) D)]$, and $\mathbf{d}$ is a $P \times 1$ vector of desired signal $\mathbf{d}(n)=[d(n), d(n-D), \ldots, d(n-(P-1) D)]^{T} . P$ must be a positive integer, and usually $P \leq M$. $C(n)$ is equal to $\mathbf{I}$, and $\mathbf{W}(n)=\left(\mathbf{X}^{T}(n) \mathbf{X}(n)\right)^{-1}$.

By substituting the parameters $P$ and $D$ and the matrices $\mathbf{C}$ and $\mathbf{W}$ from Table 1 in (2), we can acquire various types of conventional algorithms such as binormalized data reusing least mean square (BNDR-LMS), regularized APA, and the normalized LMS with orthogonal correction factor (NLMSOCF) [11] that are various types of the affine projection's family. In Table 1 , the parameter $\varepsilon$ is the regularization parameter which is introduced for preventing division by zero, and $\mu$ is the step size that controls the convergence speed and steady-state mean square error.

If the filter coefficient equation is updated only once every $K$ iterations, it is named partial rank algorithm (PRA) [12].

2.2. SR-APA. The filter coefficients update equation for SRAPA is given by [13]

$$
\mathbf{h}(n+1)=\mathbf{h}(n)+\mu \mathbf{X}_{G}(n)\left(\mathbf{X}_{G}^{T}(n) \mathbf{X}_{G}(n)\right)^{-1} \mathbf{e}_{G}(n),
$$

where $\mathbf{e}_{G}(n)=\mathbf{d}_{G}(n)-\mathbf{X}_{G}^{T}(n) \mathbf{h}(n)$.

$G=\left\{i_{1}, i_{2}, \ldots, i_{q}\right\}$ denotes a $q$-subset (subset with $q$ members) of the set $\{0,1, \ldots, P-1\}$, and also $\mathbf{X}_{G}(n)$ and $\mathbf{d}_{G}(n)$
TABLE 1: Family of APA adaptive filter algorithms.

\begin{tabular}{lcccc}
\hline Algorithm & $P$ & $D$ & $\mathbf{C}(n)$ & $\mathbf{D}(n)$ \\
\hline APA & $P \leq M$ & $D=1$ & $\mathbf{I}$ & $\left(\mathbf{X}^{T}(n) \mathbf{X}(n)\right)^{-1}$ \\
BNDR-LMS & $P=2$ & $D=1$ & $\mathbf{I}$ & $\left(\mathbf{X}^{T}(n) \mathbf{X}(n)\right)^{-1}$ \\
R-APA & $P \leq M$ & $D=1$ & $\mathbf{I}$ & $\left(\varepsilon \mathbf{I}+\mathbf{X}^{T}(n) \mathbf{X}(n)\right)^{-1}$ \\
NLMS-OCF & $P \leq M$ & $D \geq 1$ & $\mathbf{I}$ & $\left(\mathbf{X}^{T}(n) \mathbf{X}(n)\right)^{-1}$ \\
\hline
\end{tabular}

are the $M \times q$ matrix of the input signal and the $q \times 1$ vector of the desired signal, respectively. They are defined as

$$
\begin{aligned}
& \mathbf{X}_{G}(n)=\left[\mathbf{x}\left(n-i_{1} D\right), \mathbf{x}\left(n-i_{2} D\right), \ldots, \mathbf{x}\left(n-i_{q} D\right)\right], \\
& \mathbf{d}_{G}(n)=\left[d\left(n-i_{1} D\right), d\left(n-i_{2} D\right), \ldots, d\left(n-i_{q} D\right)\right]^{T} .
\end{aligned}
$$

The indices of $G$ are obtained by the following procedure.

(1) Compute the following values for $0 \leq i \leq P-1$ :

$$
\frac{e^{2}(n-i D)}{\|\mathbf{x}(n-i D)\|^{2}},
$$

where $\mathbf{e}(n)=[e(n), e(n-D), \ldots, e(n-(P-1) D)]^{T}$.

(2) Compute $q$ largest values of (5) that corresponded to indices of $\mathrm{G}$.

Setting $D=1$ leads to SR-APA presented in [13]. By substituting the parameters $D$ and $P$, various types of SR-APA and SR-BNDR-LMS, SR-NLMS-OCF can be established. The filter coefficient update for SR-APA can be represented as

$$
\begin{aligned}
\mathbf{h}(n+1)= & \mathbf{h}(n)+\mu \mathbf{X}(n) \mathbf{B}(n) \\
& \times\left(\mathbf{B}^{T}(n) \mathbf{X}^{T}(n) \mathbf{X}(n) \mathbf{B}(n)\right)^{-1} \mathbf{B}^{T}(n) \mathbf{e}(n),
\end{aligned}
$$

where $\mathbf{B}(n)=\left\{\mathbf{1}_{i 1}, \mathbf{1}_{i 2}, \ldots, \mathbf{1}_{i q}\right\}$ is the $P \times q$ matrix and $\mathbf{1}_{i q}=$ $[0, \ldots, 0,1,0, \ldots, 0]$ is the $P \times 1$ vector with the element 1 in the position $i_{q}$.

2.3. $S P U-A P A$. We use the Lagrange multiplier method to solve the following optimization problem [6]:

$$
\operatorname{Min}\left\|\mathbf{h}_{F}(n+1)-\mathbf{h}_{F}(n)\right\|^{2}
$$

subject to $\mathbf{d}(n)=\mathbf{X}^{T}(n) \mathbf{h}(n+1)$. Recursive equation for updating filter coefficients can be written as

$$
\mathbf{h}_{F}(n+1)=\mathbf{h}_{F}(n)+\mu \mathbf{X}_{F}(n)\left(\mathbf{X}_{F}^{T}(n) \mathbf{X}_{F}(n)\right)^{-1} \mathbf{e}(n) .
$$

$F=\left\{j_{1}, j_{2}, \ldots, j_{S}\right\}$ denotes the indices of the $S$ blocks out of $B$ blocks that should be updated at every adaptation, $\mathbf{X}_{F}(n)=$ $\left[\mathbf{X}_{j_{1}}^{T}(n), \mathbf{X}_{j_{2}}^{T}(n), \ldots, \mathbf{X}_{j_{S}}^{T}(n)\right]^{T}$ is $S L \times P$ matrix, and $\mathbf{X}_{i}(n)=$ $\left[\mathbf{x}_{i}(n), \mathbf{x}_{i}(n-D), \ldots, \mathbf{x}_{i}(n-(P-1) D)\right]_{L \times P}$.

The indices of $F$ are obtained by the following procedure.

(1) Compute the following values for $1 \leq i \leq B$ :

$$
\operatorname{Tr}\left(\mathbf{X}_{i}^{T}(n) \mathbf{X}_{i}(n)\right)
$$

(2) Compute $S$ largest values of (9) that corresponded to indices of $F$. 
TABLE 2: Explanation of computational complexity of the APA, SR-APA, and SPU-APA.

\begin{tabular}{lcccc}
\hline Algorithm & Multiplications & Divisions & Additional multiplications & Comparisons \\
\hline APA & $\left(P^{2}+2 P\right) M+P^{3}+P^{2}$ & & & \\
SR-APA & $\left(q^{2}+2 q\right) M+q^{3}+q^{2}$ & $P$ & $(P-q) M+P+1$ & $P \log _{2} q+O(P)$ \\
SPU-APA & $\left(P^{2}+2 P\right) S L+P^{3}+P^{2}$ & & 1 & $B \log _{2} S+O(B)$ \\
\hline
\end{tabular}

\section{Channel Modelling}

Different types of attenuations such as scattering from surface, reflecting from bottom, frequency absorption, and noise influence the sound waves propagation and decrease the energy of wave in shallow water channel. Scattering from surface and reflecting from bottom are two important events that influence reliable transmission in underwater acoustic communication channels. For modelling of the scattering from surface, we use Rayleigh surface loss model. We use Strait of Hormuz conditions and Hamilton-Backman model for counting losses due to reflect from bottom. Loss due to frequency absorption is another type of loss that happened in underwater acoustic propagation. This phenomenon happens in some material such as magnesium sulphate $\left(\mathrm{MgSO}_{4}\right)$ and boric acid $\left(\mathrm{B}(\mathrm{OH})_{3}\right)[14,15]$.

Noise in this channel is the combination of ambient noises such as turbulences, shipping noise, thermal noise, and sea-state noise that is dependent on frequency and can be described by Gaussian statistics. For studying multipath propagation in this channel, we consider ray theory model [16] as a mathematical model. The channel impulse response which is obtained from this channel is shown in Figure 1. We can conclude that after sixth-path channel pattern we have strong attenuation, so there is not a signal reception. We can consider sixth-path channel pattern for this channel.

\section{Computational Complexity}

The computational complexity of the introduced algorithms is shown in Table 2. This table shows the number of multiplications, divisions, and comparisons at each iteration. The computational complexity of APA and that of SRAPA are from [13]. By comparing computational complexity between APA and SPU-APA, we can see the reduction in multiplications equal to $\left(P^{2}+2 P\right)(M-S L)$. Also, SPU-APA needs 1 additional multiplication and comparison $B \log _{2} S+$ $O(B)$.

\section{Simulation and Results}

In the channel model, the channel depth is 40 meters. Transmitter and receiver are placed in depth of 5 and 10 meters from the surface, respectively, and the distance between them is $1 \mathrm{Km}$. We use QPSK modulation with bandwidth of $5 \mathrm{KHz}$, carrier frequency of $23 \mathrm{KHz}$, and SNR of $20 \mathrm{~dB}$. Also, channel characteristics are based on the data measured in the Strait of Hormuz by NOAA submarine during August of 2009.

We use the structure of Figure 2 for equalizer. In this structure, we have two modes, training and testing modes

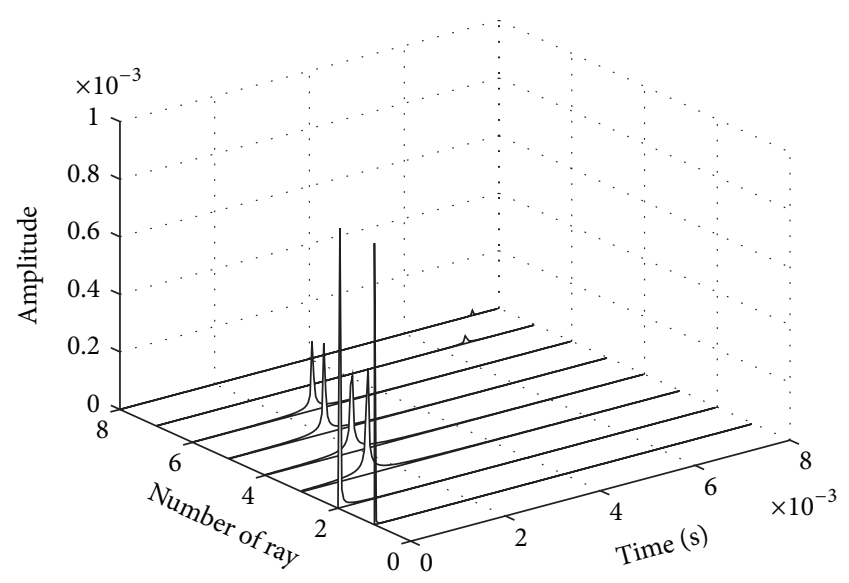

FIGURE 1: Impulse response of channel used in this paper.

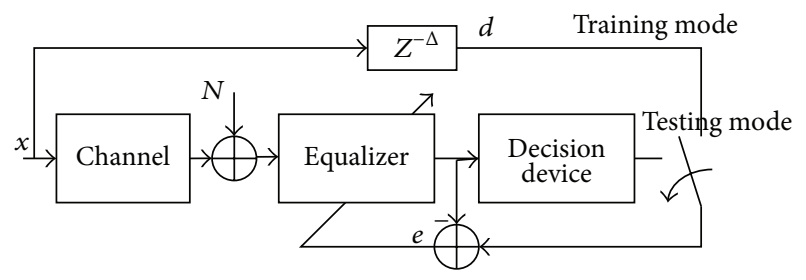

FIgURE 2: Structure of an adaptive channel equalizer.

with 2500 and 5000 samples, respectively, and also the number of filter taps is 240 and $\Delta=120$. For minimizing bit error rate, the step sizes of LMS, NLMS, and APA were set to $10^{-9}, 0.5$, and 0.1 , and the order of APA was equal to 4 . In SR-APA, $q$ parameter is the number of input regressors that have been selected. It can be changed from 1 to 4 . In SPU-APA algorithm, the number of total blocks is 4 , and $S$ denotes the number of blocks that must be selected by (9).

Figure 3 shows the learning curve of LMS, APA, and SRAPA. We can observe that APA and SR-APA have faster convergence rate than LMS. Figure 4 shows the learning curve of LMS, APA, and SPU-APA. The SR-APA and SPU-APA have close convergence rate to APA with lower computational complexity than APA. In comparison with LMS, the steadystate mean square error (MSE) of SR-APA by selecting 3 of 4 input regressors decreases by $5.8(\mathrm{~dB})$, and the steadystate MSE of SPU-APA by selecting 3 of 4 blocks of filter coefficients decreases by $5.5(\mathrm{~dB})$.

Symbol error rate (SER) curves versus signal-to-noise ratio have been shown in Figures 5 and 6 for APA, SPU-APA, 


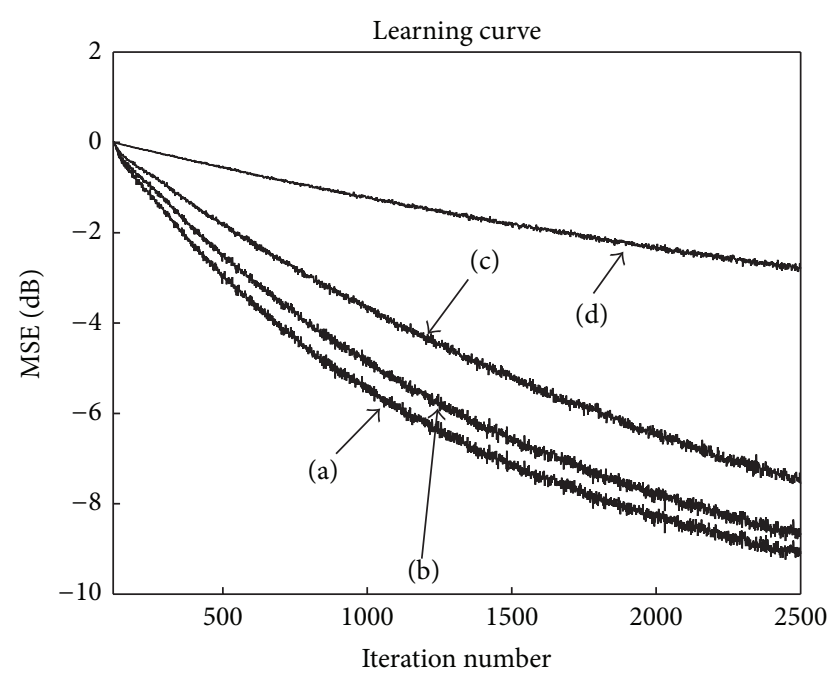

(a) APA: $P=4$

(b) SR-APA: $P=4$ and $q=3$

(c) SR-APA: $P=4$ and $q=2$

FIGURE 3: Learning curves for the LMS, APA, and SR-APA with $\mu=$ $10^{-9}$ for LMS and $\mu=0.1$ and $P=4$ for APA and SR-APA.

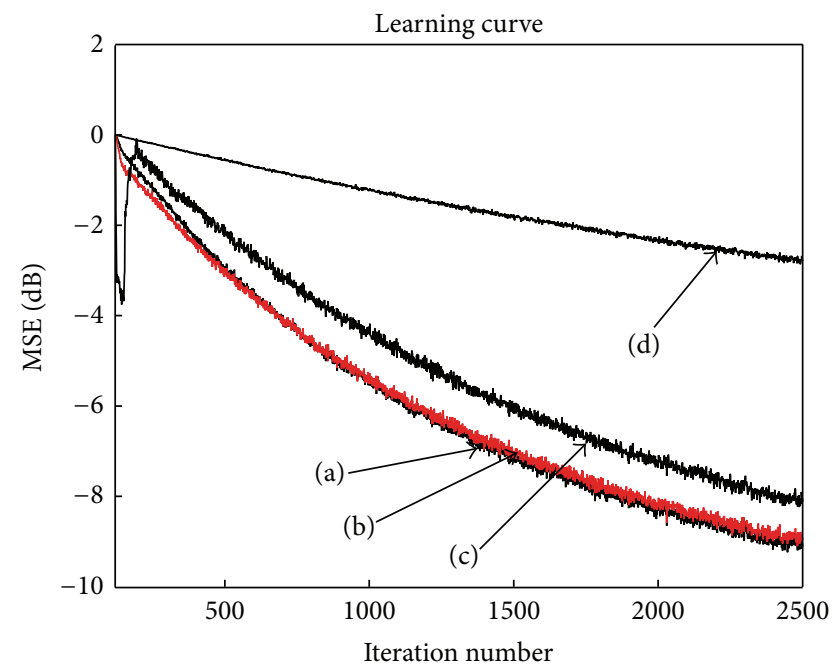

(a) APA: $P=4$

(b) SPU-APA: $P=4, B=4$ and $S=3$

(c) SPU-APA: $P=4, B=4$ and $S=2$

(d) LMS

FIGURE 4: Learning curves for the LMS, APA, and SPU-APA with $\mu$ $=10^{-9}$ for LMS, $\mu=0.1$ and $P=4$ for APA, and $\mu=0.1, P=4, B=4$, and $S=2,3$ for SPU-APA.

and SR-APA, respectively. The SER of SR-APA and SPU-APA is $10 e-2$, while the SER of LMS is $10 e-1$ at SNR of $20(\mathrm{~dB})$.

\section{Conclusion}

In this paper, we have applied selective regressor affine projection algorithm (SR-APA) and selective partial update APA (SPU-APA) in shallow water channel. Also, we have compared the performances of these algorithms with the

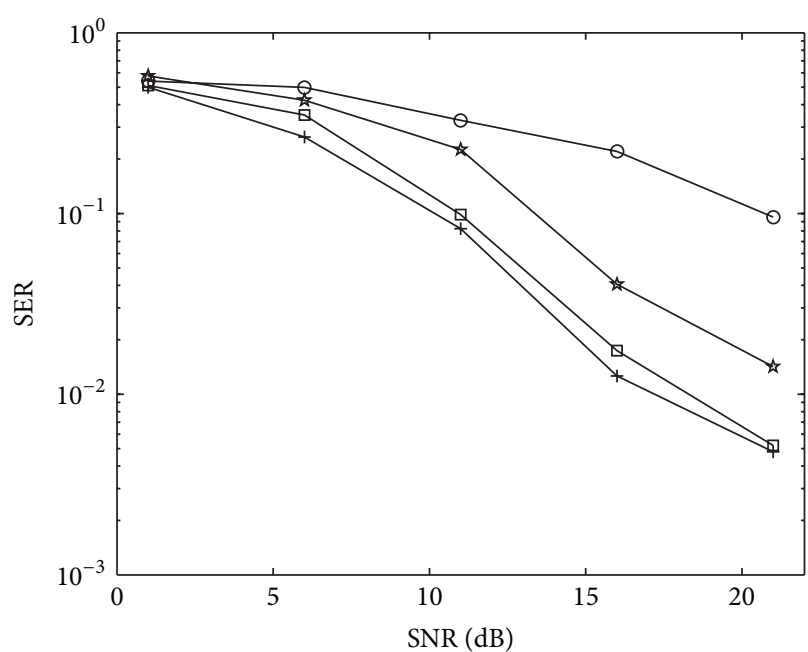

$$
\begin{aligned}
& \leftarrow \text { APA: } P=4 \\
& \square \text { SPU-APA: } P=4, B=4 \text { and } S=3 \\
& \square \text { SPU-APA: } P=4, B=4 \text { and } S=2 \\
& \neg \text { LMS }
\end{aligned}
$$

FIGURE 5: Symbol error rate versus SNR for APA and SPU-APA.

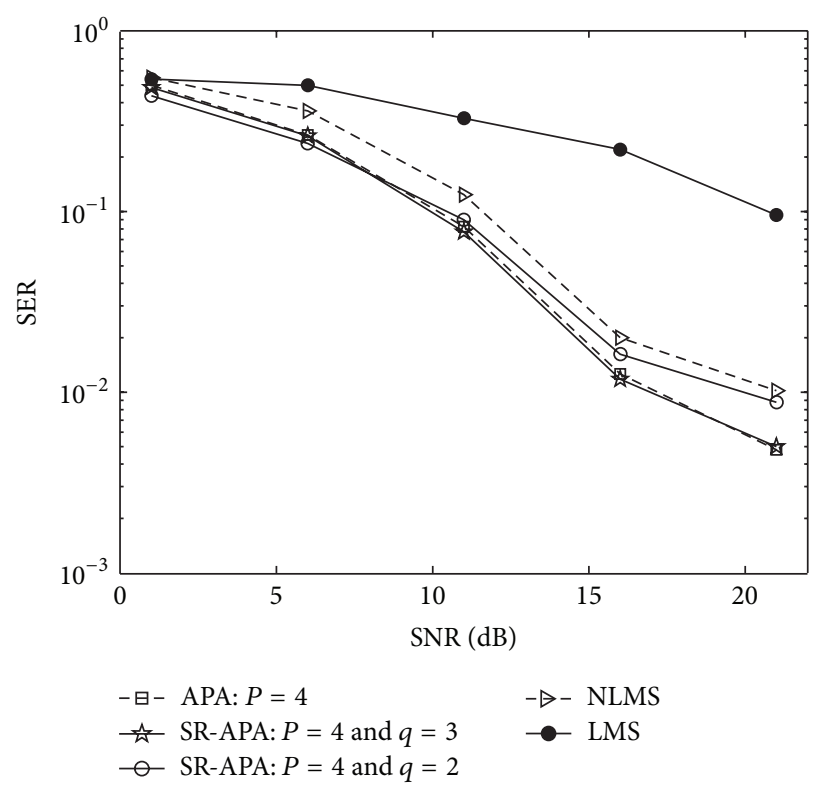

FIGURE 6: Symbol error rate versus SNR for APA and SR-APA.

LMS and classical affine projection algorithm. These algorithms have good tradeoff between convergence rate and steady-state mean square error in comparison with APA algorithms.

\section{Conflict of Interests}

The authors declare that there is no conflict of interests regarding the publication of this paper. 


\section{References}

[1] M. Stojanovic, J. A. Catipovic, and J. G. Proakis, "Phasecoherent digital communications for underwater acoustic channels," IEEE Journal of Oceanic Engineering, vol. 19, no. 1, pp. 100$111,1994$.

[2] M. Stojanovic, J. Catipovic, and J. G. Proakis, "Adaptive multichannel combining and equalization for underwater acoustic communications," Journal of the Acoustical Society of America, vol. 94, no. 3, pp. 1621-1631, 1993.

[3] Y. R. Zheng, C. Xiao, T. C. Yang, and W.-B. Yang, "Frequencydomain channel estimation and equalization for shallow-water acoustic communications," Physical Communication, vol. 3, no. 1, pp. 48-63, 2010.

[4] L. Freitag, M. Johnson, and M. Stojanovic, "Efficient equalizer update algorithms for acoustic communication channels of varying complexity," in Proceedings of the IEEE Conference on Oceans, pp. 580-585, October 1997.

[5] T. Schertler, "Selective block update of NLMS type algorithms," in Proceedings of the International Conference on Acoustics, Speech and Signal Processing (ICASSP'98), pp. 1717-1720, Seattle, Wash, USA, May 1998.

[6] K. Dogançay and O. Tanrikulu, "Adaptive filtering algorithms with selective partial updates," IEEE Transactions on Circuits and Systems II: Analog and Digital Signal Processing, vol. 48, no. 8, pp. 762-769, 2001.

[7] S. Werner, M. L. R. de Campos, and P. S. R. Diniz, "Partialupdate NLMS algorithms with data-selective updating," IEEE Transactions on Signal Processing, vol. 52, no. 4, pp. 938-949, 2004.

[8] S. C. Douglas, "Analysis and implementation of the max-NLMS adaptive filter," in Proceedings of the 29th Asimolar Conference on Signals, Systems and Computers, pp. 659-663, Pacific Grove, Calif, USA, October 1995.

[9] T. Aboulnasr and K. Mayyas, "Selective coefficient update of gradient-based adaptive algorithms," in Proceedings of the International Conference on Acoustics, Speech, and Signal Processing, pp. 1929-1932, Munich, Germany, April 1997.

[10] G. L. Sicuranza and A. Carini, "Filtered-X affine projection algorithm for multichannel active noise control using secondorder Volterra filters," IEEE Signal Processing Letters, vol. 11, no. 11, pp. 853-857, 2004.

[11] S. G. Sankaran and A. A. Beex, "Normalized LMS algorithm with orthogonal correction factors," in Proceedings of the 31st Asilomar Conference on Signals, Systems \& Computers, pp. 16701673, November 1997.

[12] S. G. Kratzer and D. R. Morgan, "The partial-rank algorithm for adaptive beamforming," in Real-Time Signal Processing VIII, vol. 0564 of Proceedings of SPIE, pp. 9-14, 1985.

[13] K.-Y. Hwang and W.-J. Song, "An affine projection adaptive filtering algorithm with selective regressors," IEEE Transactions on Circuits and Systems II: Express Briefs, vol. 54, no. 1, pp. 4346, 2007.

[14] H. Medwin and C. S. Clay, Fundamentals of Acoustical Oceanography, Academic Press, San Diego, Calif, USA, 1998.

[15] R. P. Hodges, Underwater Acoustics Analysis, Design and Performance of Sonar, John Wiley \& Sons, New York, NY, USA.

[16] L. M. Brekhovskikh and Y. Lysanov, Fundamentals of Ocean Acoustics, Springer, Berlin, Germany, 3rd edition, 2003. 

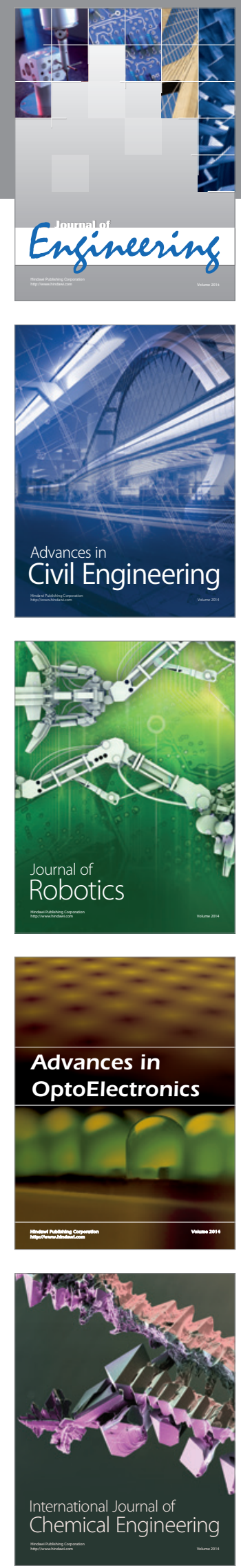

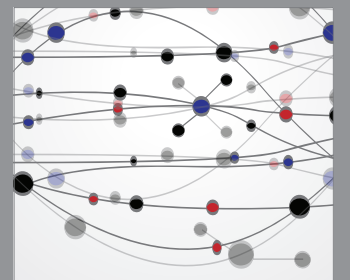

The Scientific World Journal
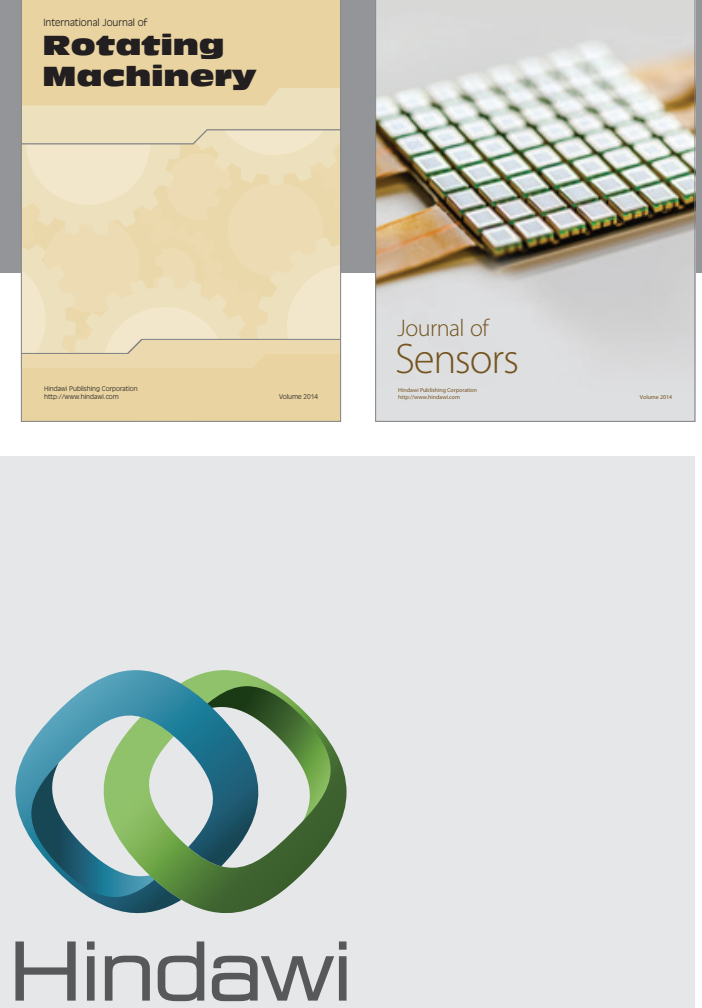

Submit your manuscripts at http://www.hindawi.com
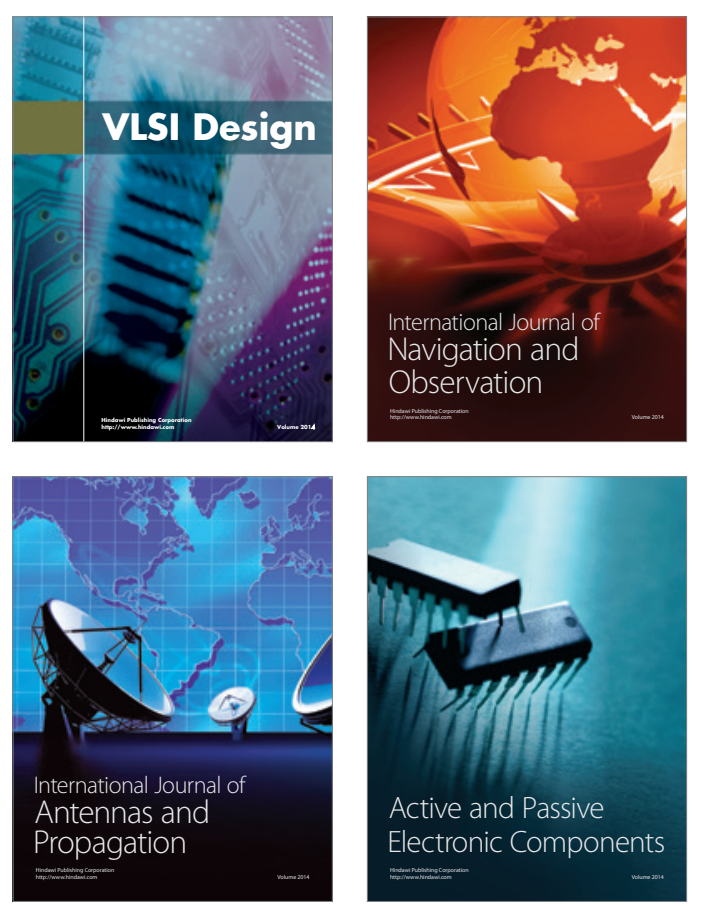
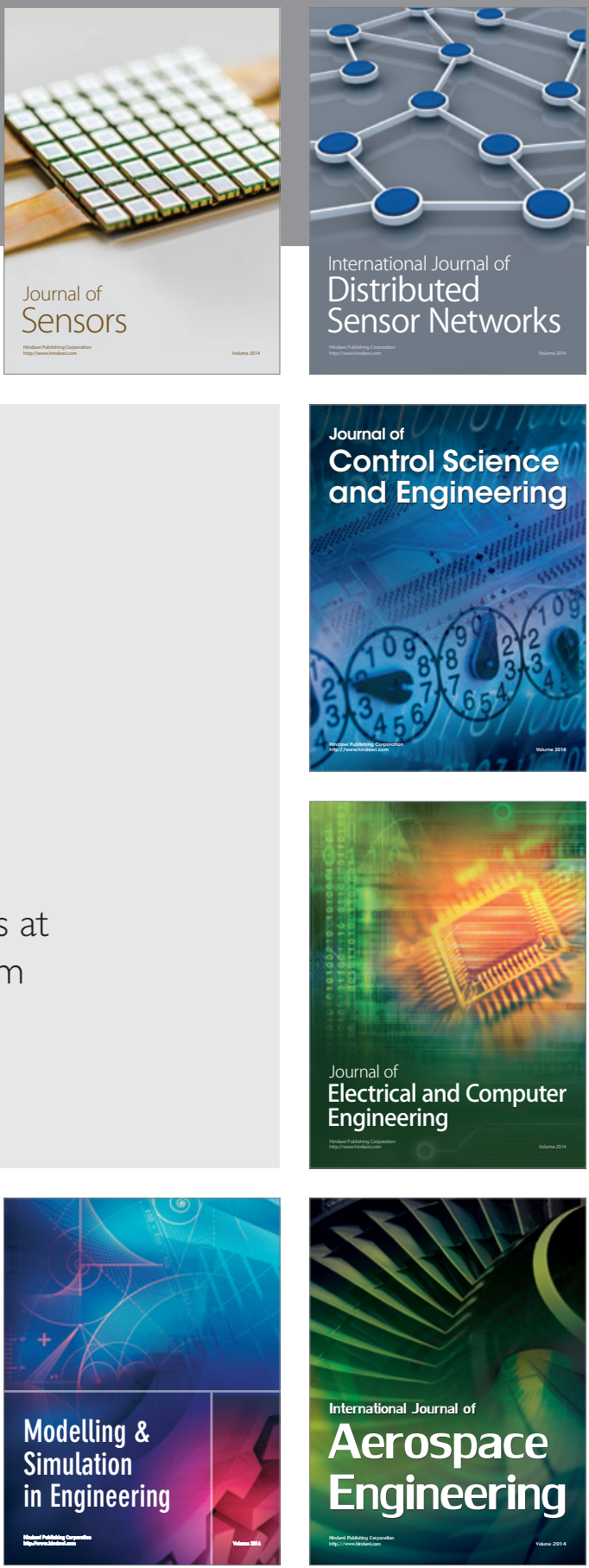

Journal of

Control Science

and Engineering
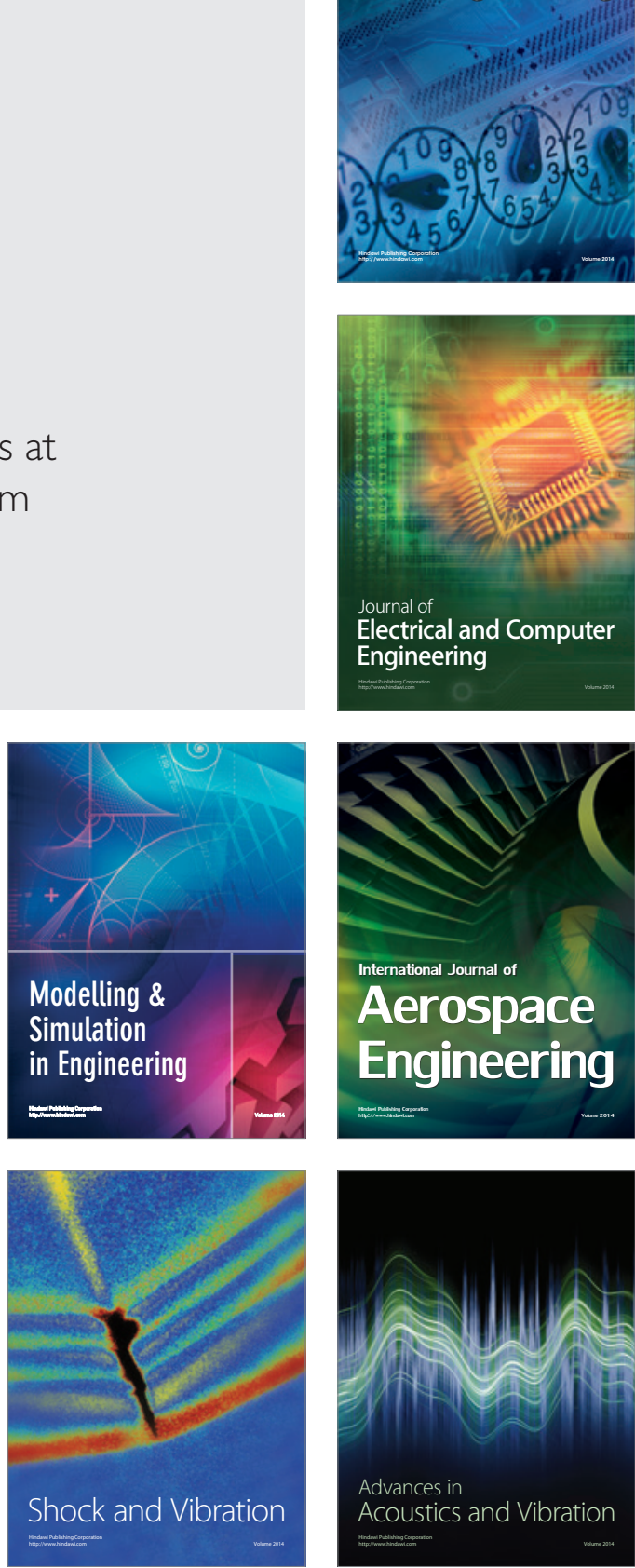